\title{
WIMP Searches with Liquid Xenon: the XENON10 Experiment
}

\author{
Laura Baudis ${ }^{* \dagger}$ \\ University of Florida, USA \\ E-mail: lbaudis@ufl.edu
}

The XENON experiment searches for Weakly Interacting Massive Particles (WIMPs) with liquid xenon (LXe) as the active target. The detector is a 3-D position sensitive Time Projection Chamber optimized to simultaneously measure the ionization and scintillation produced by a recoil event down to energies of $16 \mathrm{keV}$. The distinct ratio of the two signals for nuclear recoils arising from WIMPs and neutrons and for electron recoils from the dominant gamma-ray background determines its event-by-event discrimination. With 1 ton of LXe distributed in ten identical modules, the proposed XENON1T will achieve a sensitivity more than a factor of thousand beyond current limits. A phased program will test the $10 \mathrm{~kg}$ target (XENON10) followed by a $100 \mathrm{~kg}$ (XENON100) module. I review the progress of the XENON R\&D phase before presenting the status of XENON10. The experiment will be based at the Gran Sasso Underground Laboratory and is expected to start data taking in 2006.

International Europhysics Conference on High Energy Physics

July 21st - 27th 2005

Lisboa, Portugal

\footnotetext{
*Speaker.

${ }^{\dagger}$ for the XENON Collaboration
} 


\section{Introduction}

Weakly Interacting Massive Particles (WIMPs) populating the halo of our galaxy can be detected directly, via their interactions with nuclei in terrestrial detectors, or indirectly, via their annihilation products in the galactic center, halo, or sun [1]. XENON is a new generation dark matter detector, with the aim of observing the small energy released after a WIMP scatters off a Xe nucleus [2]. Using a target mass of 1 ton, it proposes to reach a sensitivity of $10^{-46} \mathrm{~cm}^{2}$ for spin-independent cross sections, which is several orders of magnitude below the current best limit of $1.6 \times 10^{-43} \mathrm{~cm}^{2}$ [3]. Discrimination of signal and background is based on the distinct ratio of the ionization and scintillation signals produced in LXe by nuclear (from WIMPs and neutrons) and electron (from gamma, beta and alpha backgrounds) recoil events [4]. The challenge is to accomplish the eventby-event discrimination down to $16 \mathrm{keV}$ nuclear recoil energy in a large volume detector. The background will be additionally suppressed by passive gamma and neutron shields and by the detector's 3-D position resolution. To verify achievable thresholds, background rejection power and sensitivity, a $10 \mathrm{~kg}$ prototype (XENON10) is being developed for deployment at the Gran Sasso Underground Laboratory (3500 mwe). The goal of XENON10 is to reach a sensitivity of 2 dark matter events $/ 10 \mathrm{~kg} /$ month, without the need of a muon veto for fast neutrons. XENON10 will also pave the way for the design of a $100 \mathrm{~kg}$ detector. With 3 months of operation underground, at a background level below $1 \times 10^{-5}$ events $\mathrm{kg}^{-1} \mathrm{~d}^{-1} \mathrm{keV}^{-1}$ after rejection, XENON100 will achieve a sensitivity of $10^{-45} \mathrm{~cm}^{2}$ (see Fig. 1). XENON1T will be realized with ten identical XENON100 modules.

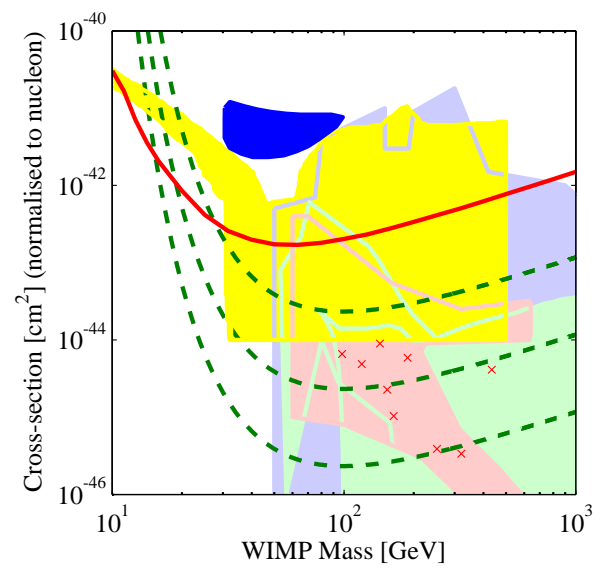

Figure 1: Experimental results and theoretical predictions for spin-independent WIMP nucleon cross sections. The dark filled region shows the DAMA 3- $\sigma$ signal region [5], the solid line is the current CDMS limit [3]. The dotted lines are projected sensitivities for XENON10, XENON100 and XENON1T [2]. Theoretical predictions are from [8]. Figure generated with limitplotter [dmtools.brown.edu].

\section{Results from Prototype Dual Phase Detectors}

Several prototypes, with Xe mass ranging from few hundred grams to few kilograms, have been designed to test the feasibility of the concept and measure detector characteristics such as 
energy threshold, background discrimination as well as the scintillation and ionization efficiency of nuclear recoils in liquid Xe. Some results from this R\&D work have been previously reported [6]. A $7.1 \mathrm{~cm}^{3}$ Xe chamber with 2 Hamamatsu R9288 PMTs optimized for Xe light was built to study the scintillation efficiency of Xe recoils as a function of energy and electric field. The scintillation yield for recoils of energies between 10.4 to $56.5 \mathrm{keV}$ (Fig. 2, left), and for applied fields up to 4 $\mathrm{kV} / \mathrm{cm}$ was measured with a nearly mono-energetic neutron beam with an average energy of 2.4 $\mathrm{MeV}$ [7]. The ionization yield of low energy nuclear recoils in LXe and the background rejection power (see Fig. 2, right) of a dual phase xenon chamber, was studied with a detector equipped with one PMT in the liquid and one in the gas, irradiated with a $5 \mathrm{Ci}^{241} \mathrm{AmBe}$ neutron source. The absolute number of ionization electrons from nuclear recoils was measured in the energy range of 10-100 keV. Contrary to expectations, the charge yield of nuclear recoils in LXe was found to be surprisingly high and with a very weak electric field dependence. Even more surprising, the yield increases with decreasing recoil energy. Results from these experiments are being finalized for publication.
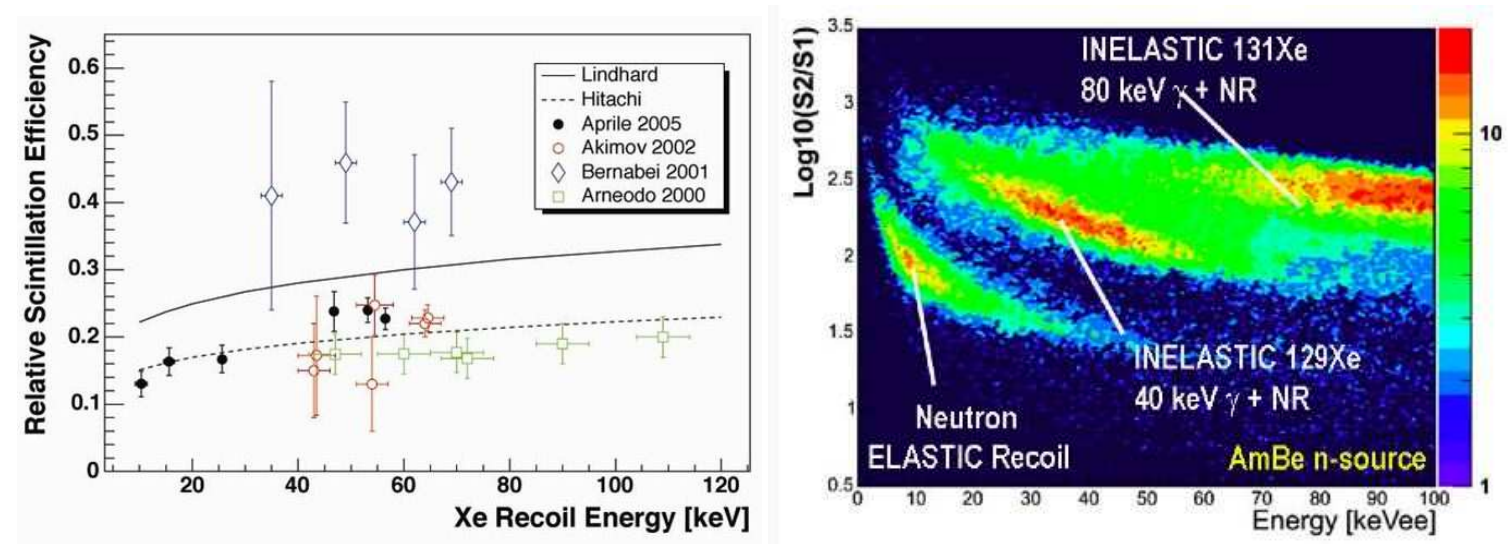

Figure 2: Scintillation yield of low energy nuclear recoils (left) and discrimination power of low energy nuclear recoils (right) studied with smaller prototypes.

Figure 3 (left) shows the design of the XENON10 module. It is a dual phase TPC, with an active mass of $10 \mathrm{~kg}$ contained in a drift region in the liquid of $20 \mathrm{~cm}$ length. An array of 1 inch square, metal channel low radioactivity UV sensitive PMTs (Hamamatsy R8520-AL), 48 in the gas and 32 in the liquid, detect both primary and secondary signals from an event. Since the proportional light is produced in a small spot with the same X-Y coordinates as the primary interaction site, 2-D localization with better than $1 \mathrm{~cm}$ position resolution is feasible. Drift time measurements will provide full 3-D localization information, allowing background reduction via fiducial volume cuts. The 3-D position sensitivity of a dual phase XeTPC has been demonstrated with a dual phase chamber with $10 \mathrm{~cm}$ drift gap, equipped with 21 PMTs in the gas and 14 PMTs in the liquid (see Fig. 3, right). Studies with this detector, irradiated with ${ }^{252} \mathrm{Cf}$ neutrons and 2.5 MeV neutrons from a D-D generator, have been recently carried out and analysis of these data is ongoing. Preliminary results show excellent discrimination power down to a threshold of $10 \mathrm{keV}$ recoil energy.

The expected XENON10 background was simulated in detail using a GEANT4 based Monte Carlo code. The dominant contribution comes from the ${ }^{238} \mathrm{U}$ and ${ }^{232} \mathrm{Th}$ chains in the PMT arrays. 

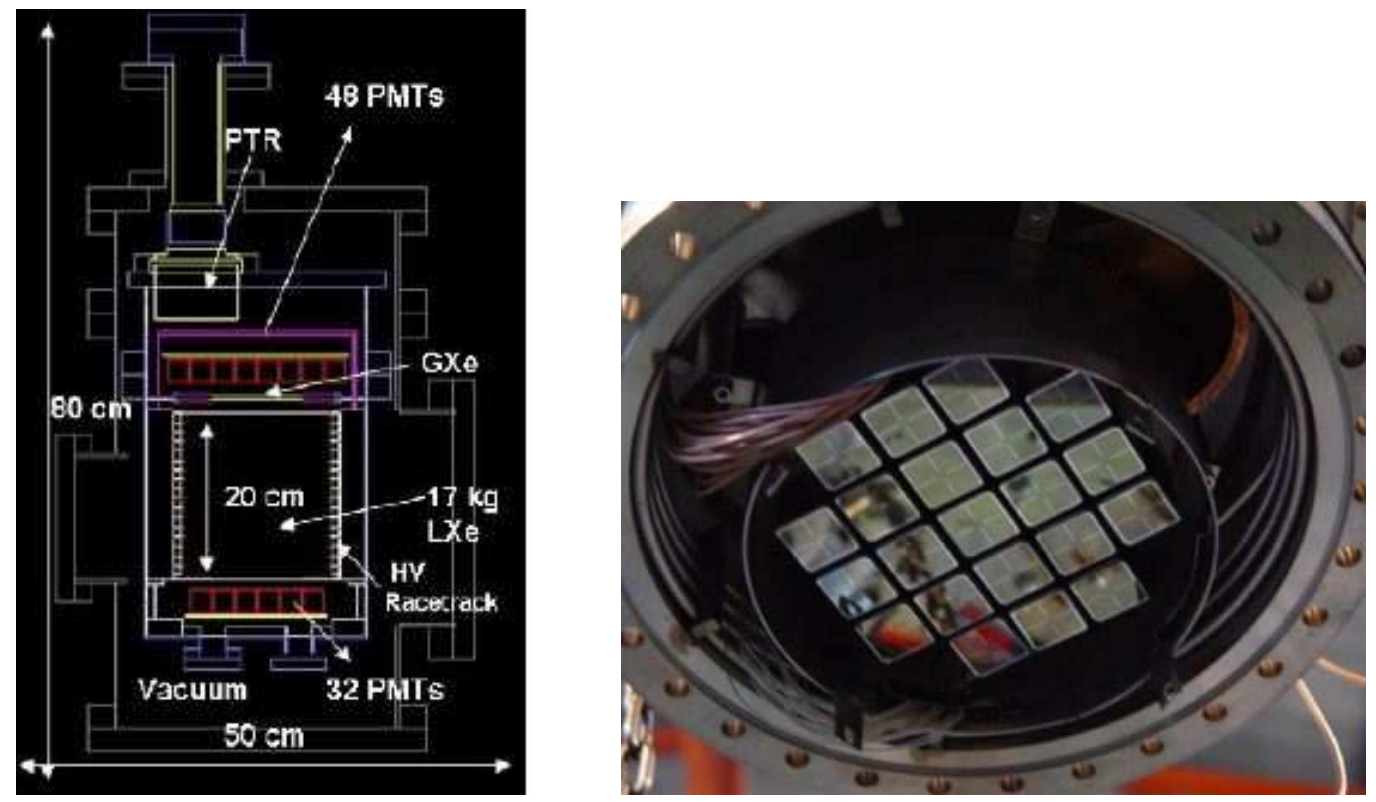

Figure 3: Schematic of the XENON10 detector module (left) and a picture of a 21 PMT array (right).

It can be reduced to the desired level with a fiducial volume cut leaving $\sim 10.3 \mathrm{~kg}$ of active LXe mass, from a total of $17.3 \mathrm{~kg}$ of LXe. A material screening program is underway using two highpurity Ge (HPGe) spectrometers in appropriate shielding at the Soudan Mine. The $2 \mathrm{~kg}$ and $500 \mathrm{~g}$ HPGe detectors are screening the detector and shield components (such as the stainless steel, teflon, PMTs, etc) for their U/Th/K/Co content. The results will be used for a full Monte Carlo model of the XENON10 background, and will guide the design of the first $100 \mathrm{~kg}$ module. At present, the XENON10 cryostat, the gas handling/purification and cryogenics systems, the slow control system as well as the electronics and DAQ system are already built and tested with the 21/14 PMTs detector. The larger inner chamber with 46/32 PMTs will be of similar design. It is expected that the experiment will be installed in the Gran Sasso Laboratory at the beginning of 2006.

\section{References}

[1] K. Freese, astro-ph/0508279, L. Baudis, astro-ph/0511805.

[2] XENON Collaboration XENON: A Liquid Xenon Experiment for Dark Matter Proposal submitted to NSF, Particle and Nuclear Astrophysics, Proposal 0201740 (September 2001).

[3] CDMS Collaboration, astro-ph/0509259, accepted in PRL (2005)

[4] M. Yamashita et al. Astropart. Phys. 20, 79 (2003).

[5] R. Bernabei et al.., Phys. Lett. B 424, 195 (1998).

[6] E. Aprile et al., astro-ph/0502279.

[7] E. Aprile et al. Phys. Rev. D 72072006 (2005).

[8] H. Baer et al., JCAP 0309, 007 (2003), E. Baltz and P. Gondolo, Phys. Rev. D 67, 063503 (2003), A. Bottino et al., Phys.Rev. D 69, 037302 (2004), J. Ellis et al., Phys.Rev. D 71095007 (2005), A. Masiero, S. Profumo and P. Ullio, Nucl. Phys. B 712 86-114 (2005). 